lastly that the present diversity of speech on the globe admis of another, a much more simple and rational explanation.

What are the facts? Col. Garrick Mallery has an interesting paper also in last week's NATURE, on Gesture Speech, in which be tells us that there are, or were, in the United States alone sixty-five stock languages differing from each other "as radically as each differed from the Hebrew, Chinese, or English." And how many more in Mexico, Central, and South America? In Europe we have at least one still surviving, the Basque. In Asia there are at least thirty-five or forty. But who will count the number in the Sudan, and in the Oceanic regions occupied by the Negrito, Papuan, and Melanesian tribes? It is no exaggera. tion to say that probably as many as two thousand of these stock languages have been evolved since man first began to utter articulate sounds.

Now if it be necessary to postulate two independent evolutions of buman speech in order to account for two independent forms of speech, it follows that we must postulate two thousand independent evolutions of human speech in order to account for these two thousand independent forms of speech. Are the advocates of speechless races prepared to go this length? Or do anthropologists at all realise the nature of the problem, when they propose to explain the existence of fundamentally distinct Janguages by the assumption of a corresponding number of independent centres of linguistic evolution? If they draw the line short of one or two thousand such centres, how do they propose to meet the difficulty presented by so many separate types of speech? Frederic Muiller left the problem just where it was when he arbitrarily fixed the number of physical and lingaistic families at twelve.

But so marvellous is the evolution of speech, that one may well doubt whether it occurred even so many as twelve time ever since the appearance of man on the earth. For my part I decline to believe that it occurred more than once, if once be sufficient to account for the present conditions. And it is on this ground that I take my stand. Anything short of, say, two thousand evolutions of speech are inadequate; one suffices: Under like conditions speech becomes differentiated far more rapidly than physical features. The former is essentially more or less evanescent; the latter are relatively persistent. Hence during the many ages of man's life on the globe, his physical type has been but slightly modified, producing mere varieties-a black and woolly-haired, a yellow and lank-haired, a fair and wavy-haired variety, and so on. But the primeval linguistic type or germ has become differentiated into varieties, species and even genera, whence the various morphological orders of speech, four in number, and the many now fundamentally distinct groups and families developed within each of those morphological orders, some extinct, some dying out, some still flourishing. The germ itself, which served as the common starting point, but which was itself at first little more than speech "in petto," has long been effaced past all recovery. Hence, although starting from one common centre, it does not follow that the linguistic families now existing can ever again be traced back to that common centre. Aided as we are by embryology and the fossil world, can we trace back the various orders of plants and animals to their common centres? Yet no evolutionist doubts that they were differentiated from such centres. But language, although it may be said to have a sort of embryology within itself, revealing the growth of its inner structure, leaves no fossils behind it. Its "missing links" are lost for ever. Hence it is not surprising that, in dealing with the evolution of speech, much more must be postulated than is always necessary in dealing with the evolution of organised life. It follows that while Darwinism as applied to organisms may one day be established scientifically, Darwinism as applied to language, must always partake somewhat of the nature of a hypothesis. Meanwhile I submit that, on the reasons here given, the hypothesis of a common primeval Iinguistic germ is both rational and adequate, whereas the hypothesis of speechless races is both improbable in itself, and fails to account for the very conditions to explain which it has been invented.

A. H. KEANE

\section{The Chemistry of the Plante and Faure Batteries}

In your iscue of the 20 th ult. there is a letter by Dr. Oliver Lodge on the recent experiments of $\mathrm{Mr}$. Tribe and myself. While confirming our general results from his own experience, he asks a question about the "lead sulphate into which we state the spongy lead is converted during the discharge of a Planté or Faure battery.

In an early stage of our investigation we satisfied ourselves that lead sulphate was capable of both oxidation and reduction by the voltaic current, under the circumstances found in these batteries. Our best experiment is described in NATuRE of March r6. It was made by spreading lead sulphate on platinum plates ; but I have just had it repeated with learl plates, so as to imitate more closely the conditions of actual practice. The sulphate was reduced by the electrolytic hydrogen as before. As, however, the reduction takes place first in close proximity to the lead plate, it is not easily recognised till the chemical change has advanced some distance, and a good deal of the white salt always escaped decomposition. But the circumstances of the actual practice are much more favourable for the reduction of the sulphate than were those of our experiment: for the sulphate is formed in perfect contact with the metallic lead of the plate or its spongy covering, and the reduction is doubtless facilitated by its intimate mixture with the excess of spongy lead, When we stated that sulphate of lead is finally the "only product of the discharge," we were referring to the disappearance of any peroxide, and did not mean to imply that in actual practice the whole of the spongy metal is usually converted into sulphate.

In our experiments Mr. Tribe and I have always employed a sufficiency of acid, and we have never found any difficulty in charging again a plate which had been discharged.

In conclusion, I may express my great satisfaction that Dr. Lodge is carrying on an independent inquiry into the obscure chemical changes that take place in these ceils.

Bowness, August 5

J. H. GLadstone

\section{The Late Prof. Balfour}

Permit me to add a few words to Dr. Foster's admirable biographical sketch in the last number of NATURE, and thereby correct a slight error into which he has fallen. He assigns to me the credit of inviting our much-lamented friend to give lectures on animal morphology. It behoves me to say that I have no claim to so much foresight. The proposal, so characteristic of Prof. Balfour's ardent disposition, originated, to the best of my belief, with him, and all I had to do was to place at his service, with the consent of the Vice-Chancellor for the time being, my private room in the New Museums, which I was glad to see turned to so good a purpose, for hitherto but little use had been made of it. The result is sufficiently well known.

44, Davies Street, London, August 5

\section{Raoul Pictet's Corpuscular Theory of Gravitation}

I BELIEVE that I can remove M. Pictet's uncertainties regard ing the credibility of the presumptive origin of attractive force in the undirected motion of an all-pervading material ether, without adopting the desponding alternative to which he appears to be obliged (in perhans needless extremities) to betake himself, that it might be conceded "without its being possible to explain it." My reasons for acce, ting the proposition without any doubt or question, would at least, I believe, if they could be submitted to him in a form of faultless coherence and comple eness, relieve him from pursuing the laborious purpose, which I am perfectly assured from my own apprehension of the real character of the equivalence, and of the mode of establishment which. it admits of, would fail in its intended object, of undertaking a series of pendulum experiments to prove it.

Before reading the translation in NATURE, vol, xxvi. p. 3 IO, of M. Pictel's paper on a comparison between the potential and corpuccular theories of attractive force, I had in fact just assured myself satisfactorily of the correctness of exactly the conclusion of which he has given such a clear and distinct enunciation, from a theory of thermodynamic actions which proceeds upon an entirely different basis from that which he has skilfully, and in so many cases successfully, applied. The demonstration which I used is a sufficiently clear and consistent one to be convincing; but it is founded upon a chain of reasoning which is quite independent of that employed by M. Pictet, and it does not actually lead me to entertain the theoretical conclusion that the apparent force of gravitation on a planet will be in any measure directly dependent on, and variable with the varying velocities of other planets' motions in the solar system; but that it will be a constant effect of the ethereal medium. If therefore the proof which I could 